\title{
11
}

\section{Error Reduction of DMPSK Transmitted Over Flat Fading Channels}

\author{
Michel Fattouche ${ }^{1}$ and Hatim Zaghloul ${ }^{2}$
}

1 The University of Calgary, Calgary, Alberta, Canada, T2N1N4

2 R\&D, AGT Limited, 19E, 411 1st St. S.E., Calgary, Alberta, Canada, T2G 4Y5

\section{ABSTRACT}

This paper proposes two algorithms for an error reduction techniqueldemodulator to minimize the effects of the propagation channel on DMPSK modulated symbols transmitted over a flat fading channel. The first algorithm uses a Hilbert transform whereas the second algorithm uses a parametric two-ray time domain model to estimate the absolute value of the phase differential of the carrier from the envelope of the carrier. This is demonstrated on a mobile radio channel.

\section{INTRODUCTION}

The North American emerging standard for digital cellular telephones (IS-54 [1]) calls for a phase modulation scheme; namely, $\pi / 4$ offset DQPSK. Phase modulation is superior to amplitude modulation since it can be applied differentially. Differential modulation is advantageous in minimizing the effect of the propagation channel on the transmitted symbols. A typical characteristic of mobile RF channels is short term fading which is the establishment of a standing wave pattern through the 
superposition of waves travelling over paths of different lengths. The nulls of this pattern occur about every half wavelength of the wave in question ( $\approx 0.175 \mathrm{~m}$ for $850 \mathrm{MHz}$ waves). If this pattern is assumed to be stationary, for simplicity, then an antenna mounted on a vehicle travelling at $27.7 \mathrm{~m} / \mathrm{s}(\approx 100 \mathrm{~km} / \mathrm{hr})$ will encounter a null approximately every $6.3 \mathrm{~ms}$. With FM modulation, this leads to FM click noise $(\approx 159 \mathrm{~Hz})$. In the case of $24.3 \mathrm{kSymbols} / \mathrm{s} \pi / 4$ offset QPSK, this noise seems irrelevant. This is not the case; the differential phase of the standing wave pattern is composed of long intervals of constant value (corresponding to intervals of constant amplitude) and short intervals of spikes [2] (corresponding to nulls in the amplitude). If the spikes are $1 \%$ of the $6.3 \mathrm{~ms}$, then the antenna will traverse the spike in 0.063 ms every 153.1 symbols. This duration is long enough to introduce a symbol error (which unfortunately would propagate to two symbols in differential modulation). Fattouche and Zaghloul [2] proposed a method for reducing these errors for $\pi / 4$ DQPSK. That method was based on the work of Fattouche and Zaghloul [3] on the use of a Hilbert transform introduced by Voelcker [4] to estimate the differential phase of a CW signal transmitted over a flat fading RF propagation channel. This paper shows how the technique of [2] as well as a simpler technique can be applied to reduce the errors introduced by the propagation channel on DMPSK signals.

\section{A NEW PHASE ESTIMATION TECHNIQUE}

Fattouche and Zaghloul [3] showed that it is possible to estimate the phase differential, $\delta \phi$, of a CW signal, $m(t)=|m(t)|$ 
$e^{j \phi(t)}$, transmitted over a flat fading channel from the envelope of the signal, $|m(t)|$, using the Hilbert transform

$$
\widehat{\delta \phi_{k}}=H\left[\ln \left|\hat{m}_{k}\right|-\ln \left|\hat{m}_{k-1}\right|\right]
$$

where the subscript denotes a particular sample and $\hat{\chi}$ denotes the estimated value of $\chi$. They showed that the same relationship can be used to estimate the group delay from the amplitude of the frequency response of a frequency selective channel. Fattouche and Zaghloul [2] showed that it is possible to use this formula to reduce the effect of the propagation channel on $\pi / 4$ offset DQPSK modulated symbols transmitted over a flat fading channel. We will now show a new technique of phase estimation of signals transmitted over fading channels. This is demonstrated on a CW signal transmitted over a flat fading channels. The same technique can be applied to estimate the group delay from the amplitude of the frequency response of a frequency selective channel. This technique is based on that if a bandlimited signal, $\mathbf{m}(\mathbf{t})$, is periodic, it can be represented by its Fourier series expansion as follows,

$$
\mathrm{m}(\mathrm{t})=\sum_{k=-M_{1}}^{M_{2}} c_{k} e^{j k \omega t}
$$

where $\omega=2 \pi / T, T$ is the period of $\mathbf{m}(\mathbf{t})$, and $c_{k}$, the Fourier coefficients of $\mathbf{m}(\mathbf{t})$, are equal to $M(k T)$ for $\mathbf{k}=-M_{1}, \ldots \ldots \ldots, M_{2}$. $M(f)$ is the Fourier transform of $\mathbf{m}(\mathbf{t})$ which is limited to the band $\left[-M_{1} / T, M_{2} / T\right]$. Lockhart [9] denotes $e^{j \omega t}$ by z where $t$ assumes real values only. In this paper, we adopt Lockhart's representation with a slight modification; we replace $e^{j \omega t}$ in (2) by $e^{-j \omega t}$ and obtain

$$
\mathrm{m}(\mathrm{z}) \underset{k=-M_{1}}{=} \sum_{k^{2}}^{M_{2}} c^{-k}
$$


The reason for the negative sign in $e^{-j \omega t}$ is to force $m(z)$ in (3) to become the z-transform of the discrete spectrum $\{M(k / T)\}_{k=-M_{1}}^{M_{2}}$ of $\mathrm{m}(\mathrm{t})$. From the fundamental theorem of algebra, $\mathrm{m}(\mathrm{z})$ can be factorized as

$$
m(z)=c_{-M_{1}} z^{M_{1}} \prod_{\mathrm{k}=1}^{\mathrm{N}}\left(1-\alpha_{k} z^{-1}\right)
$$

where $\alpha_{k}$ is the kth z-domain zero and $N=M_{1}+M_{2}$. Substituting $e^{-j \omega t}$ for $\mathrm{z}$ in $\mathrm{m}(\mathrm{z})$ in (4), i.e., evaluating $\mathrm{m}(\mathrm{z})$ around the unit circle in a clockwise manner, leads to

$$
m(t)=c_{-M_{1}} e^{-j M_{1} \omega t} \prod_{\mathrm{k}=1}^{\mathrm{N}}\left(1-\alpha_{k} e^{j \omega\left(t-\tau_{k}\right)}\right)
$$

where $\left|\alpha_{k}\right|$ and $\left(-\omega \tau_{k}\right)$ are the amplitude and phase of $\alpha_{k}$ respectively.

Based on (5), one can say that each z-domain zero, $\alpha_{k} \mathrm{e}^{-\omega \tau_{k}}$, of $\mathrm{m}(\mathrm{z})$ causes $\mathrm{m}(\mathrm{t})$ to fade over a time interval $t_{1}<t<t_{2}$ where $t_{1}<$ $\tau_{k}<t_{2}$. In other words, m(f) can be modelled as the sum of two rays as follows.

$$
\mathrm{m}(\mathrm{t}) \approx A_{k} e^{-j \omega^{\prime} t}\left(1-\alpha_{k} e^{j 2 \pi t / T}\right) \quad t_{1}<t<t_{2}
$$

where $\omega^{\prime}$ is a constant and $A_{k}$ is slowly time varying. This leads to

$$
\begin{aligned}
& |m(t)| \approx A_{k} \sqrt{\left(1+\left|\alpha_{k}\right|^{2}\right)-2\left|\alpha_{k}\right| \cos \omega t^{\prime}} \\
& \phi(t) \approx \tan ^{-1}\left[\frac{-\left|\alpha_{k}\right| \sin \omega t^{\prime}}{1-\left|\alpha_{k}\right| \cos \omega t^{\prime}}\right]-\omega^{\prime} t^{\prime}
\end{aligned}
$$

where $t^{\prime}=t-t_{\min }$. and $t_{\min }$ is the time at which the envelope of $\mathrm{m}(\mathrm{t})$, $\operatorname{lm}(t) \mid$ reaches its minimum during $\left[t_{1}, t_{2}\right]$. With the approximation that $\boldsymbol{t}^{\prime}$ is small, we can show that

$$
\widehat{d \phi} \frac{1}{d t} \approx \pm \frac{-1}{t_{0}} \frac{1}{1+\left(t^{\prime} / t_{0}\right)^{2}}-\omega^{\prime}
$$


where $t_{0}=\frac{|1-| \alpha_{k} \mid}{\sqrt{\left|\alpha_{k}\right|} \omega}$ is the time interval between the maximum and the minimum of $d \ln |m(t)| / d t$. The positive sign in (9) corresponds to a minimum phase fade whereas the negative sign corresponds to a maximum phase fade. The presence of \pm in (9) leads to a sign ambiguity when this equation is used to estimate the phase differential of a signal from its envelope.

\section{ERROR REDUCTION ALGORITHM}

The complex envelope, $z_{0}(t)$, of a DMPSK signal transmitted over a flat fading channel can be written as the complex envelope of the DMPSK signal $u(t)$, multiplied by the complex envelope $\mathrm{m}(\mathrm{t})$ of a $\mathrm{CW}$ signal transmitted over a flat fading channel and contaminated with additive white Gaussian noise (AWGN). Mathematically, we have

$$
z_{0}(t)=u(t) m(t)+n_{0}(t)
$$

where $\quad u(t)=\sqrt{E_{b} / T_{0}} \exp \left[j \sum_{i=-\infty}^{\infty}(2 \pi / M) \gamma_{i} p\left(t-i T_{0}\right)\right]$

$\gamma_{i}=\beta_{i}+\gamma_{i-1}, \beta_{i}$ is the information digit transmitted once every $T_{0}$ and takes one of the $M$ values in the alphabet $(0,1, \ldots \mathrm{M}-1), p(t)$ is a shaping pulse, $E_{b}$ is the average transmitted bit energy and no(t) is the zero mean white Gaussian noise.

The proposed algorithm consists of five steps. The first step consists of obtaining an estimate $|\widehat{m}(t)|$ of the envelope $|m(t)|$. This is accomplished by first obtaining the envelope $\left|z_{0}(t)\right|$ of $z_{0}(t)$, then bandlimiting it to twice the Doppler bandwidth, i.e. to the frequency 
band $\left[-2 f_{d}, 2 f_{d}\right]$. This bandlimitation reduces the power of the thermal noise which is directly related to the signal bandwidth. We bandlimit $\left|z_{0}(t)\right|$ to $2 f_{d}$ because the envelope of a bandlimited signal occupies twice the signal's bandwidth. As an example, let us assume that an IS-54 signal is transmitted over a mobile radio flat fading channel with relative velocity between the transmitter and the receiver of $100 \mathrm{Km} / \mathrm{Hr}$ and with a wavelength $\lambda$ of $0.28669 \mathrm{~m}$. In this case, $z_{0}(t)$ is assigned a $30 \mathrm{KHz}$ bandwidth and the Doppler bandwidth is $193.8 \mathrm{~Hz}$, implying that $\mathrm{m}(\mathrm{t})$ is contained within 193.8 $\mathrm{Hz}$, its envelope $|m(t)|$ is contained within $387.6 \mathrm{~Hz}$ and the noise reduction due to bandlimitation $\left|z_{0}(t)\right|$ is $10 . \log 10(30 \mathrm{KHz} / 387.6 \mathrm{~Hz})$ $\approx 19 d B$. In other words, when the received DMPSK signal-tonoise ration is $16 d B$, the received $\mathrm{m}(\mathrm{t})$ signal-to-noise ratio is $16+$ $19=35 d B$ which corresponds to a noise level with little or no effect on either (1) or (5), as will be shown in Fig-2.

The second step consists of obtaining an estimate for $\delta \phi_{k}$ using either (1) or (9), i.e. obtaining either

or

$$
\begin{aligned}
\hat{\delta \phi_{k}} & =H\left[\ln \left|\widehat{m}_{k}\right|-\ln \left|\widehat{m}_{k-1}\right|\right] \\
\hat{\delta \phi_{k}} & =\frac{1}{\widehat{t_{0}}} \frac{-1}{1+\left(\left(k T_{0}-\hat{t}_{\min }\right) / \hat{t}_{0}\right)^{2}}
\end{aligned}
$$

where $\widehat{m}_{k}=\widehat{m}\left(k T_{0}\right)$. In equation $(12 \mathrm{~b}), \delta \phi_{k}$ is obtained over the segments $\left[k_{1}, k_{2}\right]$ corresponding to the intervals $\left[t_{1}, t_{2}\right]$ where $|\widehat{m}(t)|$ fades at least $3 d B$ less than its running mean, i.e. $k_{1}=t_{1} / T_{0}$ and $k_{2}$ $=t_{2} / T_{0}$. 
The third step consists of estimating the mean $\mu$ of $\delta \phi_{k}$. This is achieved by obtaining the estimate $\hat{\mu}$ of $\mu$ where

$$
\widehat{\mu}=\frac{1}{N} \sum_{\mathrm{k}=1}^{N}\left(\angle z_{0, k}-\angle z_{0, k-1}\right)
$$

and $\angle z_{0, k}$ is the phase of $z_{0, k}=z_{0}\left(k T_{0}\right)$.

The fourth step consists of estimating the sign of $\delta \phi_{k}$. This is achieved by computing the two values

$$
\begin{aligned}
& S_{+}=\sum_{k=k_{1}}^{k=k_{2}} \min _{\alpha_{k} \in\{0, \ldots, M-1\}}\left\{\left(\angle z_{0, k}-\angle z_{0, k-1}\right)-\left|\hat{\delta \phi_{k}}\right|-\gamma_{k}\right\}^{2} \\
& S_{-}=\sum_{k=k_{1}}^{k=k_{2}} \min _{\alpha_{k} \in\left\{0_{0}, \ldots, M-1\right\}}\left\{\left(\angle z_{0, k}-\angle z_{0, k-1}\right)+\left|\hat{\delta \phi_{k}}\right|-\gamma_{k}\right\}^{2}
\end{aligned}
$$

if $S_{+} \mathrm{c} S$ - then $\delta \hat{\phi_{k}}+\widehat{\mu}$ in (13a\&b) is assigned a positive sign, otherwise, it is assigned a negative sign.

The fifth step consists of adjusting for the phase differential due to the flat fading channel. This is achieved by subtracting $\left(\widehat{\delta \phi_{k}}+\widehat{\mu}\right)$ from $\left(\angle z_{0, k}-\angle z_{0, k-1}\right)$. One has to note that the third and fourth steps in the above algorithm attempt to remove the two discrepancies associated with estimates (12a) and (12b); namely the nonzero bias in $\widehat{\delta \phi}_{k}$ and its sign ambiguity.

One also has to note that when a square-root Nyquist pulse is employed in the transmitter to shape the DMPSK signal (such as in IS-54) $z_{0, k}$ has to be match-filtered first before any decision is made, and unless $z_{0, k}$ is adjusted for both amplitude and phase 
distortion, match-filtering can cause Intersymbol Interference (ISI) to the DMPSK signal. For this reason, we suggest to adjust for the amplitude distortion due to the flat fading channel by dividing $z_{0, k}$ by $\left|\hat{m}_{k}\right|$ and to adjust for the phase differential as explained above, before match-filtering $z_{0, k}$.

In this algorithm, the data record can be an IS-54 [1] TDMA frame with 159 complex symbols and 1 sample/symbol. The reason only one fade per data record is recommended is that the proposed sign determination algorithm will become less accurate and more complex if it needs to determine the sign of more than one phase differential segment per data record. Note that for a symbol rate of $24.3 \mathrm{kSymbols} / \mathrm{s}$, a TDMA frame will last $6.54 \mathrm{~ms}$. If a vehicle's speed is $100 \mathrm{~km} / \mathrm{hr}$, then it will travel approximately half a wavelength (of $850 \mathrm{MHz}$ waves) each frame. This suggests that a TDMA frame is an appropriate data record.

\section{BIT ERROR RATE CALCULATION [2]}

In order to make a decision at time $t_{0}=n T_{0}$ at the receiver for $\boldsymbol{n}=1,2$ and $\mathbf{T}=41.2 \mu \mathrm{s}$, the received DMPSK signal is first passed through a filter matched to a square-root raised cosine pulse (of rolloff 0.35 ) then sampled at $t_{0}$. Without error reduction, a decision variable, $U$, is obtained as the angle between the present sample, $z_{0, n}$, and the sample $z_{0, n-1}$ out of the matched filter (MF). With error reduction, $U$ is obtained as the angle between $z_{n}$ and $z_{n-1}$ minus (or plus) the estimated phase differential at $t_{0}$. In both cases, the probability of symbol error conditioned on having transmitted the phase $\phi_{n}$ is 1-P $\left\{U_{1} \leq U<U_{2}\right\}$ where $U_{1}=\phi_{n}-\pi / M$ and $U_{2}=\phi_{n}$ $+\pi / M$. The probability $\mathbf{P}\left\{U_{1} \leq U<U_{2}\right\}$ can be obtained from 
Pawula [6] (the case of unequal signal conditions and uncorrelated noise) by expressing $z_{0, n}$ and $z_{0, n-1}$ as two phasors: $z_{0, n}=$

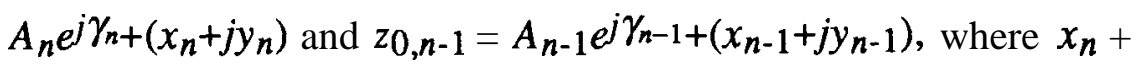
$j y_{n}$ for $n=1,2, \ldots \ldots$ and $N$ correspond to the complex AWGN out of the MF with statistical properties: $\bar{x}_{n}=\bar{y}_{n}=0$ and $\operatorname{var}\left[x_{n}\right]=$ var $\left[y_{n}\right]=\sigma^{2}$. The terms $A_{n}$ and $\alpha_{n}$ for $n=1,2, \ldots \ldots N$ are the amplitudes and phases of the distorted DMPSK signal output from the MF at time $n T_{0}$. This means that $\gamma_{n}-\gamma_{n-1}=2 \pi \beta_{n} / M+\Delta_{n}$ where $\Delta_{n}$ is the phase differential distortion introduced by the channel. The SNR $\rho_{n}$ is therefore equal to $A_{n}^{2} / 2 \sigma^{2}$ for $n=1,2, \ldots \ldots N$ and if we define $\delta \phi=\left(\gamma_{n}-\gamma_{n-1}\right) \bmod 2 \pi, U_{0}=1 / 2\left(\rho_{n}+\rho_{n-1}\right), W_{0}=$ $\sqrt{\rho_{n} \rho_{n-1}}$ and $V_{0}=1 / 2\left(\rho_{n}-\rho_{n-1}\right)$ then

$$
P\left\{U_{1} \leq U \leq U_{2}\right\}=\left\{\begin{array}{lr}
F\left(U_{2}\right)-F\left(U_{1}\right)+1 & U_{1}<\delta \phi<U_{2} \\
F\left(U_{2}\right)-F\left(U_{1}\right) & U_{1}>\delta \phi \text { or } \delta \phi>U_{2}
\end{array}\right.
$$

with: $\mathrm{F}(\mathrm{U})=\int_{-\pi 2}^{\pi / 2} d t \frac{e^{-E}}{E}\left[\frac{W_{0} \sin (\delta \phi-U)}{4 \pi} \mathrm{I}\right.$

and $E=U_{0} \cdot V_{0} \sin (?)-W_{0} \cos (\delta \phi-U) \cos (t)$.

\section{RESULTS AND CONCLUSIONS}

We will now demonstrate the use of equations (1) and (9) to estimate the phase differential of a CW signal transmitted over a flat fading channel and to reduce the effects of the channel on DMPSK modulated symbols transmitted over a flat fading channel. The channel used is the same as that of Fattouche and Zaghloul [2]. The impulse response of a mobile radio channel was measured as a 
function of distance by Bultitude [7]. Scott [8] calculated the envelope and phase of a $\mathrm{CW}$ signal transmitted over this channel as a function of distance. A block of the generated envelope is shown in Figure la versus the travelled distance. Figures 1b\&1c shows the corresponding true phase differential calculated by Scott [8] and the phase differential estimated through equation (12a) and (12b)

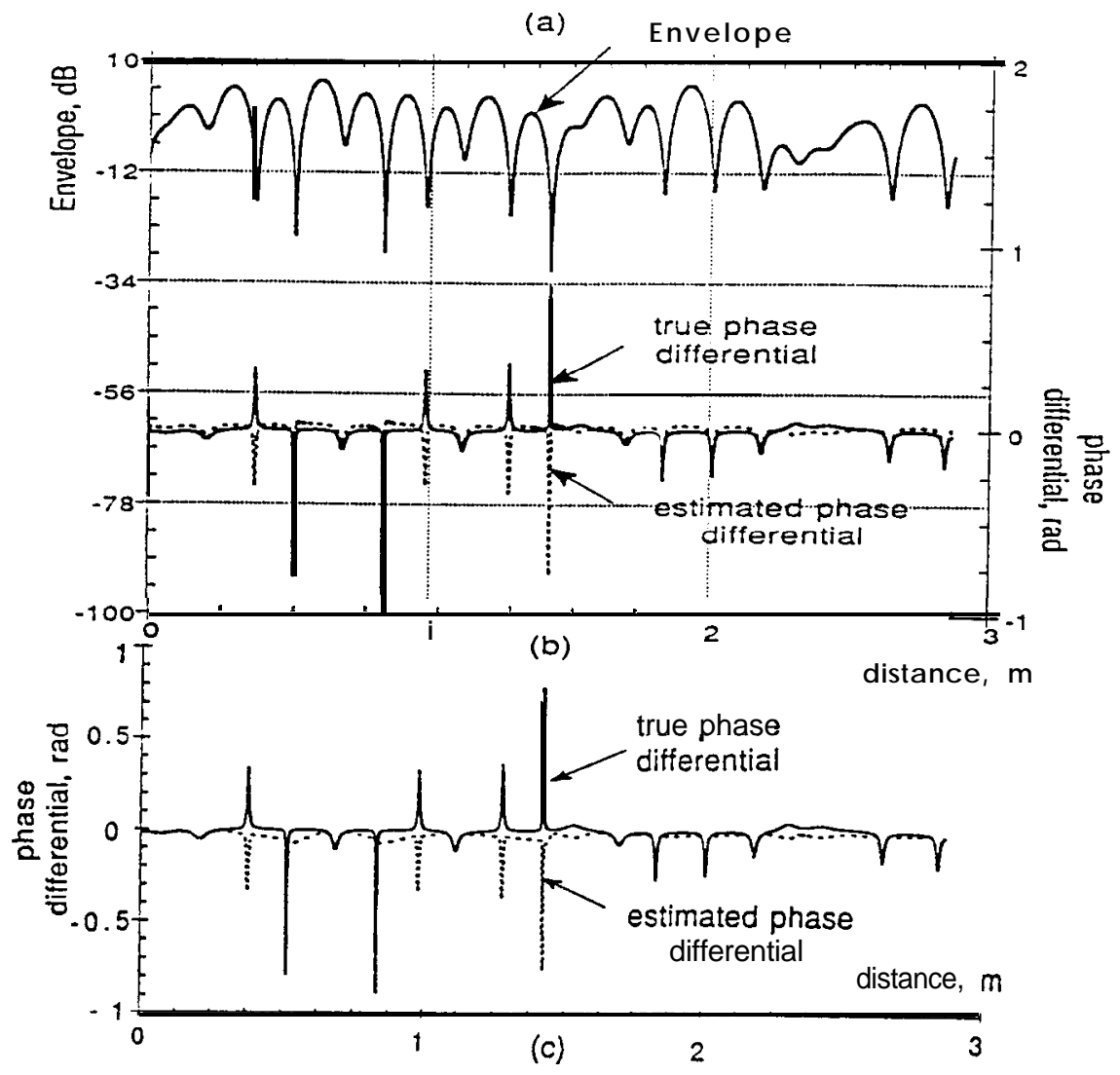

Figure 1(a) The measured envelope, (b) the measured phase differential and that estimated through (12a), and (c) the measured phase differential and that estimated through (12b). 
respectively. Three meters of this envelope were substituted into equation (14) to calculate the average BER for DMPSK modulated symbols.

In order to examine the effects of AWGN on estimates (12a) and $(12 \mathrm{~b})$, we generate a noisy signal, $m^{\prime}(t)$, from $\mathrm{m}(\mathrm{t})$ as: $m^{\prime}(t)=m(t)+n_{0}(t)$ where no(t) is a zero mean complex AWGN. The corresponding envelope and phase of $\mathrm{m}^{\prime}(\mathrm{t})$ are denoted $\left|m^{\prime}(t)\right|$ and $\phi^{\prime}(t)$ respectively. Fattouche and Zaghloul proposed that a good estimate of the goodness of fit between the estimated phase differential and the measured one is the relative mean square error, E, where

$$
E=\frac{\sum_{n=1}^{N}\left(\left|x_{n}\right|-\widehat{x_{n}} \mid\right)^{2}}{\sum_{n=1}^{N} x_{n}^{2}}
$$

Figure 2 displays E vs SNR for three cases; the first case (Case A) displays the error between the estimate $\widehat{\delta \phi_{n}}=\phi_{n}{ }^{\prime}-\phi_{n-1}{ }^{\prime}$ and $\left|\phi_{n}\right|$ The second curve (Case B) displays the error between $\left|\phi_{n}\right|$ and its unbiased estimate $\left|\hat{\delta \phi_{n}}+\widehat{\mu}\right|$ calculated using (12a). The third curve (Case C) displays the error between $\left|\phi_{k}\right|$ and its unbiased estimate $\left|\hat{\delta \phi_{n}}+\hat{\mu}\right|$ calculated using (12b) for fades that are at least 3dB less than the running mean of $\left|m^{\prime}(t)\right|$. In all three curves, $N=2047$. Two remarks can be made based on Fig-2; first, one can observe that the effects of AWGN on the estimate of $\delta \phi_{n}$ are negligible as long as the SNR is larger than $\mathbf{3 5 d B}$. Second, one can see that for SNR's larger than $35 d B$ estimate (12b) has a smaller $\mathbf{E}$ than estimate (12a). 


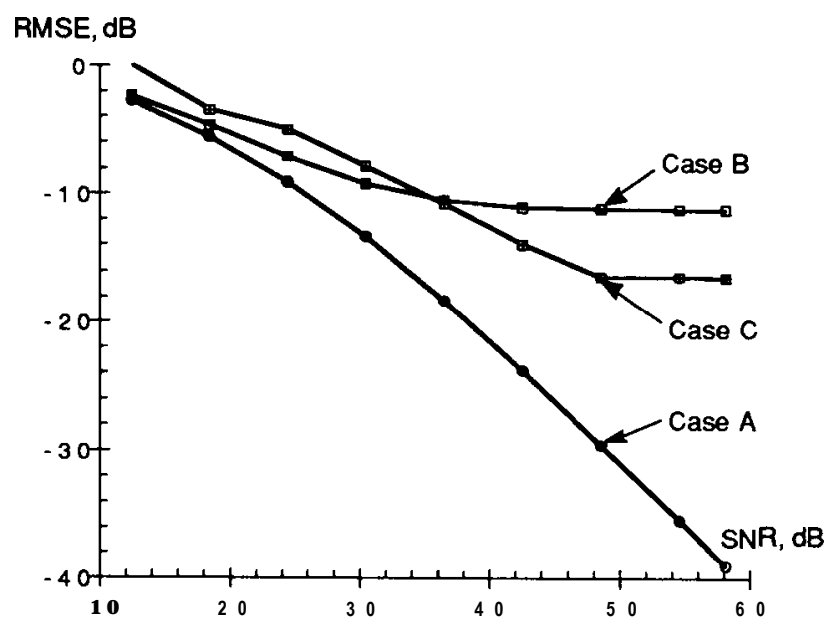

Figure 2 The error between the true phase differential and (A) its noisy version, (B) its unbiased estimation from (12a) and (C) its unbiased estimate from (12b).

In Fig-3 the BER curves are displayed versus the received DMPSK SNR per bit. The BER curves are obtained using (14). In order to turn the conditional probability into an unconditioned one, we average out $\left\{\Delta_{k}\right\}_{n=1}^{N}$ and $\left\{A_{k}\right\}_{n=1}^{N}$ over all $N$ samples as follows. When we do not adjust for the phase differential due to flat fading (case A), we substitute $\Delta_{n}$ by $\delta \phi_{n}$. When we use (12a) to adjust for the phase differential due to flat fading (case B), we substitute $\Delta k$ by $\delta \phi_{n}-\left(\delta \phi_{n}+\widehat{\mu}\right)$, where $\delta \hat{\phi_{n}}$ is obtained from (12a). When the phase adjustment is ideal (case $\mathrm{C}$ ), we set $\Delta_{n}$ equal to zero. In all three cases, we substitute for $A_{n}$ with $\left|m_{n}\right|$ for $\mathrm{N}=2047$. From Fig-3 one can see that, as previously mentioned, the irreducible BER is caused by the phase distortion due to the channel, and that by adjusting for 
the phase distortion using (12a) one can approach the limiting case where the phase distortion is entirely removed.

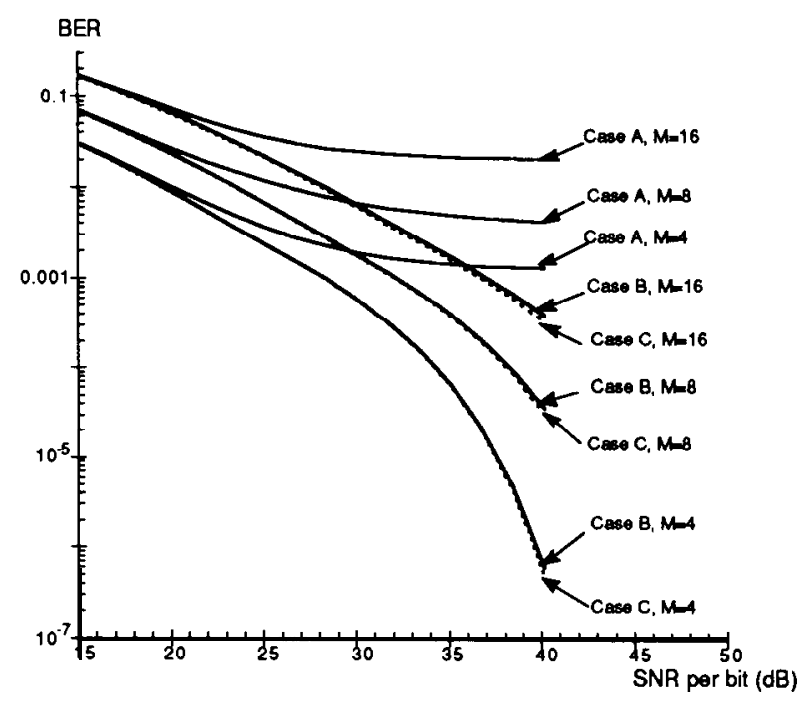

Figure 3 BER curves for $M=4,8$ and 16 each for three cases:

(A) no phase adjustment, (B) phase adjustment using equation (12a) and (C) ideal phase adjustment.

Fig-4 compares the case (A) when the adjustment for the phase distortion is accomplished using (12a) (i.e. when we replace $\Delta_{n}$ by $\left.\delta \phi_{n}-\left(\delta \phi_{n}+\widehat{\mu}\right)\right)$, with the case (B) when it is accomplished using from (12b). From Fig-4 one can see that (12b) offers a slightly better performance than (12a).

In conclusion, this paper is a direct application of the concept of estimating the phase differential of a $\mathrm{CW}$ signal transmitted over a flat fading channel using either a parametric estimate from a two-ray time domain model or a Hilbert transform. This paper proposed an 
error reduction technique/demodulator algorithm to remove the effects of flat fading channels on transmitted phase modulated digital information. The results reported in this paper suggest that the performance of the proposed error reduction technique is very close to the performance of an ideal equalizer and that the irreducible BER has been eliminated. We have developed an equalizer to eliminate the irreducible BER due frequency selective channels that operates on a similar concept.

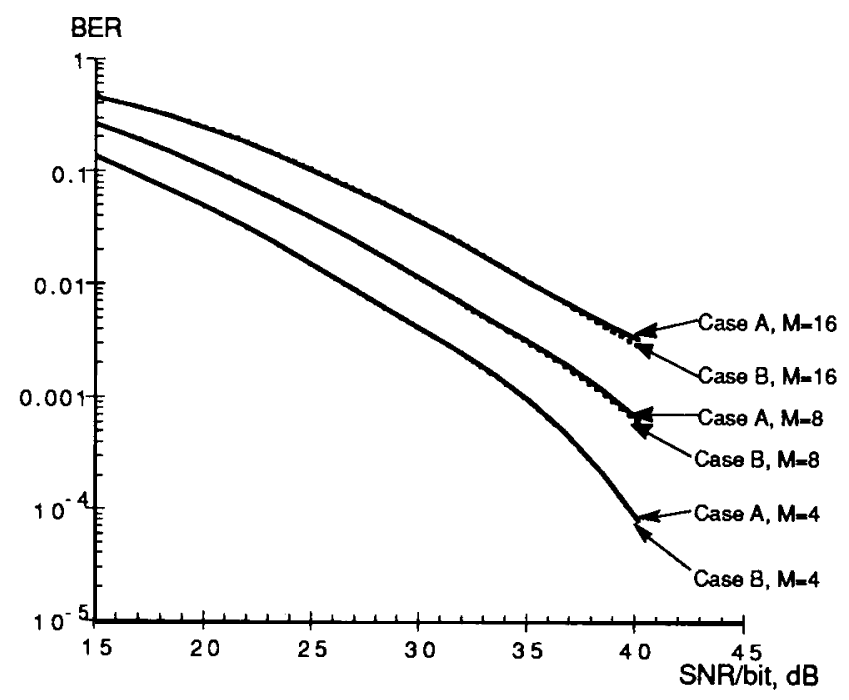

Figure 4 BER curves for $M=4,8$ and 16 for two cases:

(A) phase adjustment using equation (12a) and

(B) phase adjustment using equation (12b)

\section{REFERENCES}

[1] Cellular System, 'Dual-Mode mobile station-base station compatibility standard,' EIA/TIA, Project Number 2215, Electronic Industries Association, May 1990. 
[2] M. Fattouche, and H. Zaghloul, 'Equaliztion of $\pi / 4$ Offset DQPSK Transmitted Over Flat Fading Channels,' Proceedings of ICC'92, Chicago, IL, June 14-18, 1992.

[3] M. Fattouche, and H. Zaghloul, 'Estimation of the Phase Differential of Signals Transmitted over Fading Channels,' Electron. Lett., 27 (20), 2021-2, 1991.

[4] H. Voelcker, 'Toward a unified theory of modulation-part 1: phase-envelope relationships,' Proceedings of the IEEE, 1966, 50, pp. 340-355.

[5] H. Zaghloul, M. Fattouche and H. Zhang, 'A New Model for the Random FM and for the Group Delay of Fading Channels,' submitted to IEE Proceedings-I.

[6] R.F. Pawula, S.O. Rice, and J.H Roberts, "Distribution of the phase angle between two vectors perturbed by Gaussian noise", IEEE Trans. Comm., Vol. COM-30, pp. 1828-1841, August 1982.

[7] R.J.C. Bultitude, Measurement, characterization, and modelling of $800 / 900 \mathrm{MHz}$ mobile radio channels for digital communications, Ph.D. Dissertation, Carleton University, Feb. 1987.

[8] K.E. Scott, Adaptive Equalization and Antenna Diversity in Digital Radio, Ph.D. Dissertation, The University of Calgary, May, 1991.

[9] G.B. Lockhart, "Spectral Theory for Hybrid Modulation," IEEE Trans. Comm., Vol COM-21, pp.790-800, July 1973. 